\title{
The weil osteotomy for correction of fixed claw toe deformity and metatarsalgia
}

\begin{abstract}
Aim: The aim of our study is to evaluate the efficacy of the Weil osteotomy to correct fixed claw lesser toe deformity with dorsal subluxation or dislocation of the MTP joint and overloading metatarsalgia. Material: 120 Weil osteotomies in 60 feet of 45 patients have retrospectively been studied with a minimum follow-up of 12 months (mean f.u. 17 months). Thirty-five patients were women and ten men. For the evaluation of the result we used the AOFAS grading scale. Anteroposterior and lateral weight bearing radiographs were taken preoperatively and at the time of follow-up. Forty patients had a correction of hallux valgus deformity although 5 of the lesser toe only. All patients received a PIP fusion for the correction of the claw deformity. Results: The subjective result was at their expectation for 39 patients and beyond their expectation for 5. One patient was dissatisfied. The AOFAS score improved from 45 preoperatively to 89 postoperatively. Thirty-nine patients did not complain of metatrsalgia although for five patients metatarsalgia improved and for 1 remained at preoperative level. Shoe wearing improved in all 42 patients although for 3 this was below their expectations. Conclusion: The Weil osteotomy combined with PIP fusion is capable of correcting stiff claw lesser toe deformities with dorsal subluxation or dislocation of the MTP joint and significantly improve metatarsalgia.
\end{abstract}

Volume 3 Issue I - 2015

\author{
Evangelou $\mathrm{E}$ \\ Foot and Ankle department, Metropolitan Hospital, Greece
}

Correspondence: Evangelou E, Foot and Ankle department, Metropolitan Hospital,Athens-Greece, Tel 210480 7102, Email vagevangelou@gmail.com

Received: January 09, 2015 | Published: August 29, 2015

\section{Introduction}

The Weil osteotomy has become a popular technique for the surgical treatment of fixed claw toe deformity and metatarsalgia. The clinical examination of the patient can determine the cause of this endity, as plantar soft tissue lessions, neuroma, trauma and secondary from osteoarthritis, iatrogenic, foot structure and other etiology.

The surgical treatment of metatarsalgia has been somewhat controversial. Ideally, conservative treatment should be exhausted before surgical options are considered. Many osteotomies described as surgical solutions of the problem combined with soft tissue repair for the clawing. The Weil osteotomy has replaced a number of preceeding osteotomies by its ability to shorten the metatarsal head without elevation or depression of the metatarsal head. The shortening and transverse plane position can be carefully controlled intraoperatively. The osteotomy is usually stabilized with a solitary screw driven from dorsal to plantar in the metatarsal head. The advantage to this osteotomy is the decompression of the metatarsophalangeal joint and the resultant relaxation of the surrounding soft tissues. Generally the osteotomy is most often used on the second metatarsal. However, the same considerations including length patterns, transverse plane deformities, subluxations, and intractable plantar skin lesions sometime merit the osteotomy to be performed on the third and fourth metatarsal.

A $2 \mathrm{~cm}$ incision is made usually over the distal part of the metatarsal ending a half $\mathrm{cm}$ over the MTP joint. The extensor tendons are then retracted laterally and the dorsal periosteum and capsule are incised with the toe dorsi flexed to protect the articular cartilage. The collateral ligaments are partially incised to fully expose the metatarsal head. Usually we release totally the extensor brevis tendon the osteotomy is started $2 \mathrm{~mm}$ inferior to the most dorsal aspect of the articular cartilage at an approximately $25^{\circ}$ angle with respect to the metatarsal shaft. The angle of the osteotomy must increase toward the more lateral metatarsals because they are less plantar flexed than the second metatarsal. A solitary screw driven from proximal dorsal to distal plantar into the metatarsal head is usually sufficient. There are a multitude of different screws that can be used for this purpose including the twist-off screw. In our study we evaluated the efficacy of the Weil osteotomy to correct fixed claw lesser toe deformity with dorsal subluxation or dislocation of the MTP joint and overloading metatarsalgia. A hundred and twenty Weil osteotomies in 60 feet of 45 patients have retrospectively been studied with a minimum follow-up of 12 months (mean f.u. 17 months). Thirty-five patients were women and ten men. For the evaluation of the result we used the AOFAS grading scale. Anteroposterior and lateral weight bearing radiographs were taken preoperatively and at the time of follow-up. Forty patients had a correction of hallux valgus deformity although 5 of the lesser toe only. All patients received a PIP fusion for the correction of the claw deformity. I usually perform a proximal interphalangeal joint arthrodesis combined with a Weil osteotomy when indicated to fix the clawing of the lesser toes. The arthrodesis should be mildly flexed by bending the $\mathrm{K}$-wire after it is inserted into the toe.

Postoperatively, most patients are maintained partial weight bearing in a postoperative shoe for 7 days and then free weight bearing for 2 weeks. There was no use of crutches. The subjective result was at their expectation for 39 patients and beyond their expectation for 5 . One patient was dissatisfied. The AOFAS score improved from 45 preoperatively to 89 postoperatively. Thirty-nine patients did not complain of metatrsalgia although for five patients metatarsalgia improved and for 1 remained at preoperative level. Shoe wearing improved in all 42 patients although for 3 this was below their 
expectations. Possible complications of the Weil osteotomy include a dorsiflexed toe, pain on MTP joint dorsiflexion, reduced MTP joint range of motion, proximal interphalangeal joint contracture stiffness and a floppy toe.

The Weil osteotomy combined with PIP fusion is capable of correcting stiff claw lesser toe deformities with dorsal subluxation or dislocation of the MTP joint and significantly improve metatarsalgia. Ancillary procedures directed at correcting coexistent pathology must be considered in compound deformities.

\section{Acknowledgments}

None.

\section{Conflicts of interest}

The authors declare there is no conflict of interest. 